\title{
Introduction to the workshop
}

\author{
Jean Clairambault ${ }^{1,3, a}$ and Vitaly Volpert ${ }^{2,4, b}$ \\ ${ }^{1}$ INRIA Paris-Rocquencourt, Team Mamba, BP 105, 78153 Le Chesnay cedex, France \\ ${ }^{2}$ Institut Camille Jordan, UMR 5208 CNRS, University Lyon 1, 69622 Villeurbanne, France \\ ${ }^{3}$ Laboratoire Jacques-Louis Lions, case courrier 187, UPMC, 4, place Jussieu, 75252 Paris cedex 05, France \\ ${ }^{4}$ INRIA Team Dracula, INRIA Antenne Lyon la Doua, 69603 Villeurbanne, France
}

\begin{abstract}
Multiscale and hybrid models are complementary and are amenable to propose a coherent vision of intracellular physiology and cell population fate.
\end{abstract}

\section{Multi-scale models in cell and cell population biology}

Multi-scale models in cell biology are actively developed in order to describe physiological processes. There are various approaches to multi-scale modelling and numerous applications. Cell populations in multi-scale models can be described by discrete or continuous methods. Among them are cellular automata, lattice Boltzmann methods and various particle methods. These approaches allow a detailed description of cell behaviour, cell-cell interactions and other aspects of complex biological media. On the other hand, such individual-based models are applicable for a relatively small number of cells and they do not admit analytical investigation. Continuous models are represented by ordinary and partial differential equations for cell concentrations. In particular, these include reaction-diffusion equations, which take into account random motion of cells, and their birth and death. Navier-Stokes equations and Darcy equations describe convective motion of the medium. Other models include physiologically structured integro-differential equations, in particular to describe nonlocal non-spatial evolution phenomena in cell populations, such as carcinogenesis and drug resistance in cancer.

\section{Intracellular physiology and cell population fate}

Multi-scale models involve intracellular and extracellular regulations of biological cells. Intracellular regulation is particularly important because it determines cell fate, that is the choice between its proliferation (self-renewal), [de-]differentiation or senescence/apoptosis. Cell fate can be described by ordinary differential equations for intracellular concentrations of determinant proteins, by Boolean approaches or by probabilistic methods at the cell population level when a small number of molecules participate in this regulation and when their concentrations cannot be considered. Extracellular regulation can be effectuated by various local mechanisms in a given tissue (growth factors, cytokines) or by means of global control from other organs and tissues through endocrine signalling. Extracellular

\footnotetext{
ae-mail: jean.clairambault@inria.fr

be-mail: volpert@math.univ-lyon1.fr
} 
substances diffuse in the tissue and influence intracellular regulation of cells. Distribution of these substances can be described by reaction-diffusion equations when space is relevant to describe tissue evolution (which is not necessarily the general case).

\section{Hybrid models}

Cell populations, intracellular and extracellular regulations can also be modelled using hybrid discrete-continuous methods. These describe cells as individual objects with various lattice or off-lattice methods, intracellular regulatory networks with ordinary differential equations and extracellular substances with partial differential equations. Such models are developed in order to study differentiation in cell lineages, in particular for haematopoiesis and for other physiological processes. They can take into account cell motion, division, death, interactions with other cells and with the surrounding medium. Transition from hybrid to continuous models can be justified for some model examples. Multi-scale models can manifest interesting nonlinear dynamics, different from those obtained with classical reaction-diffusion equations.

This workshop has brought together scientists and teams working in multiscale modelling and hybrid models in cell biology, involving mathematicians, physicists and biologists. It has concluded the ANR "Bimod" research project (V. Volpert, J. Clairambault, P. Magal, 2011-2015) 